\title{
What is new in cardiac pacing ESC guidelines 2021?
}

\author{
Abbreviations \\ AVB: atrioventricular block \\ CRT: cardiac resynchronization therapy \\ ECG: electrocardiogram \\ HBP: His bundle pacing \\ $\mathrm{HF}$ : heart failure \\ LBBB: left bundle branch block \\ LVEF: left ventricular ejection fraction
}

During the last European Society of Cardiology Congress in 2021, the new guidelines for pacing and cardiac resynchronization therapy (CRT) were released (1). Since the last European guidelines for pacing were published in 2013 (2), there was a critical need for an update. Extensive recent research has been led in this field, and this becomes obvious when looking at the total number of pages and references (almost doubled in comparison with 2013). New sections have appeared concerning patient workout before implantation, but also concerning the new modalities of conduction system pacing, and a new section on perioperative management.

We summarize here the key points from these International guidelines.

A section has now been dedicated to the evaluation of patients with suspected or documented bradycardia. A very didactic table is shown to suggest a preferential electrocardiographic (ECG) monitoring according to symptom frequency: from 24-h Holter ECG in case of daily symptoms, to implantable loop recorder if symptoms occur less than one episode per month. Other means are cited such as external loop recorder / external patch recorder or handheld ECG recorder. Screening for sleep apnea syndrome now becomes a class I indication in patients with severe bradycardia or advanced atrioventricular block (AVB) during sleep. Another new point concerns the possibility to propose genetic testing in patients with early onset (age $<50$ years) of progressive cardiac conduction disease with a class IIA indication (level C).

Actually, no huge changes have been made concerning the classical bradycardic situations requiring pacing in these recent guidelines.

\section{Cardiac resynchronization therapy}

To summarize, CRT is recommended in patients with left ventricular ejection fraction (LVEF) $<35 \%$ and symptomatic heart failure (HF) (in sinus rhythm) despite optimal medical therapy, in the presence of a left bundle branch block (LBBB) QRS morphology duration > $150 \mathrm{~ms}$ (class I) or $>130 \mathrm{~ms}$ (class IIA indication). It becomes a class IIA indication in the presence of a nonLBBB morphology > 150 ms (class IIB if QRS duration > $130 \mathrm{~ms})$.

The two situations listed below now become a class I indication (versus IIA in the 2013 version):

- $\quad$ CRT, instead of right ventricular pacing is recommended in patients with an indication for ventricular pacing and high-degree $A V B$, and HF with reduced LVEF $<40 \%$ (including those in atrial fibrillation).

- In patients with HF and reduced LVEF, and symptomatic atrial fibrillation with uncontrolled heart rate, and who are candidates for atrioventricular junction ablation, CRT is recommended.

Address for correspondence:: Sok-Sithikun Bun, Pasteur University Hospital, Nice, France Phone: +33492037733

Fax:+37797989732 Email: sithi.bun@gmail.com

Received: 03.09.2021 Accepted: 04.09.2021

Copyright (C2021 Heart, Vessels and Transplantation

doi: $10.24969 /$ hvt.2020.268 


\section{Alternative pacing strategies}

To date based upon the last data and meta-analysis, no current evidence support systematical use of right ventricular septal pacing over apical pacing for all patients. One would suppose a significant place for Hisbundle pacing (HBP) in these recent guidelines, given the fact that this technique was already proposed as an accepted alternative to CRT (at the same level of evidence) in patients with AVB who have an indication for permanent pacing with LVEF between 36 and $50 \%$ (class IIA level B in the Heart Rhythm Society Guidelines published in 2018 (3)).

Here, HBP is only recommended in case of unsuccessful coronary sinus lead implantation (class IIA, level B indication). Left bundle branch area pacing was cited in this document, but further studies will be needed before integrating this new mode of stimulation. Leadless pacing will be reserved to the cases when no upper extremity venous access exists or in case of previous infection or in the presence of haemodialysis (class IIA, level B indication).

\section{Perioperative management}

A very practical and didactic section has been added in these new guidelines, from antibiotic prophylaxis (1-2 g of intravenous cefazolin recommended, class I indication), to skin antisepsic (using chlorhexidinealcohol instead of povidone-iodine-alcohol), and to heparin bridging contraindication.

For the first time, the experts recommend to use cephalic or axillary vein as a first choice for venous access, instead of the subclavian vein puncture (class IIA level B). When a pocket reintervention (upgrade for example) is scheduled, an antibiotic-eluting envelope may be considered (class IIB, level B indication).

Finally, this new edition of the European guidelines emphasizes the importance of symptoms-ECG correlation using the latest available devices for bradycardia detection. An important part of these recommendations was dedicated to patient education, patient-centered care and shared-decision making (for instance considering the choice between CRTpacemaker or CRT-defibrillator). Further studies will be needed to better define the place of physiological pacing modes (HBP and left bundle branch area pacing) in the next future in comparison with established sites of pacing.
Sok-Sithikun Bun, Emile Ferrari Cardiology Department, Pasteur University Hospital, Nice, France

Peer-review: Internal Conflict of interest: None to declare Authorship: S-S.B., E.F. are equally contributed to preparation of manuscript and fulfilled authorship criteria Acknowledgement and funding: None to declare

\section{References}

1. Glikson $M$, Nielsen JC, Kronborg MB, Michowitz $Y$, Auricchio A, Barbash IM, et al.; ESC Scientific Document Group. 2021 ESC Guidelines on cardiac pacing and cardiac resynchronization therapy. Eur Heart J 2021 Aug 29: ehab364.

2. Brignole $M$, Auricchio $A$, Baron-Esquivias $G$, Bordachar P, Boriani G, Breithardt OA, et al. ESC Committee for practice guidelines 2013. ESC Guidelines on cardiac pacing and cardiac resynchronization therapy: the Task force on cardiac pacing and cardiac resynchronization therapy of the European Society of Cardiology (ESC). Developed in collaboration with European Heart Rhythm Association. Eur Heart J 2013; 34: 2281-329.

3.Kusumoto FM, Schoenfeld MH, Barrett C, Edgerton JR, Ellenbogen KA, Gold MR, et al. 2018 ACC/AHA/HRS Guideline on the evaluation and management of patients with bradycardia and cardiac conduction delay: A Report of the American College of Cardiology/American Heart Association Task Force on Clinical Practice Guidelines and the Heart Rhythm Society. Circulation 2019; 140: e382-e482. 


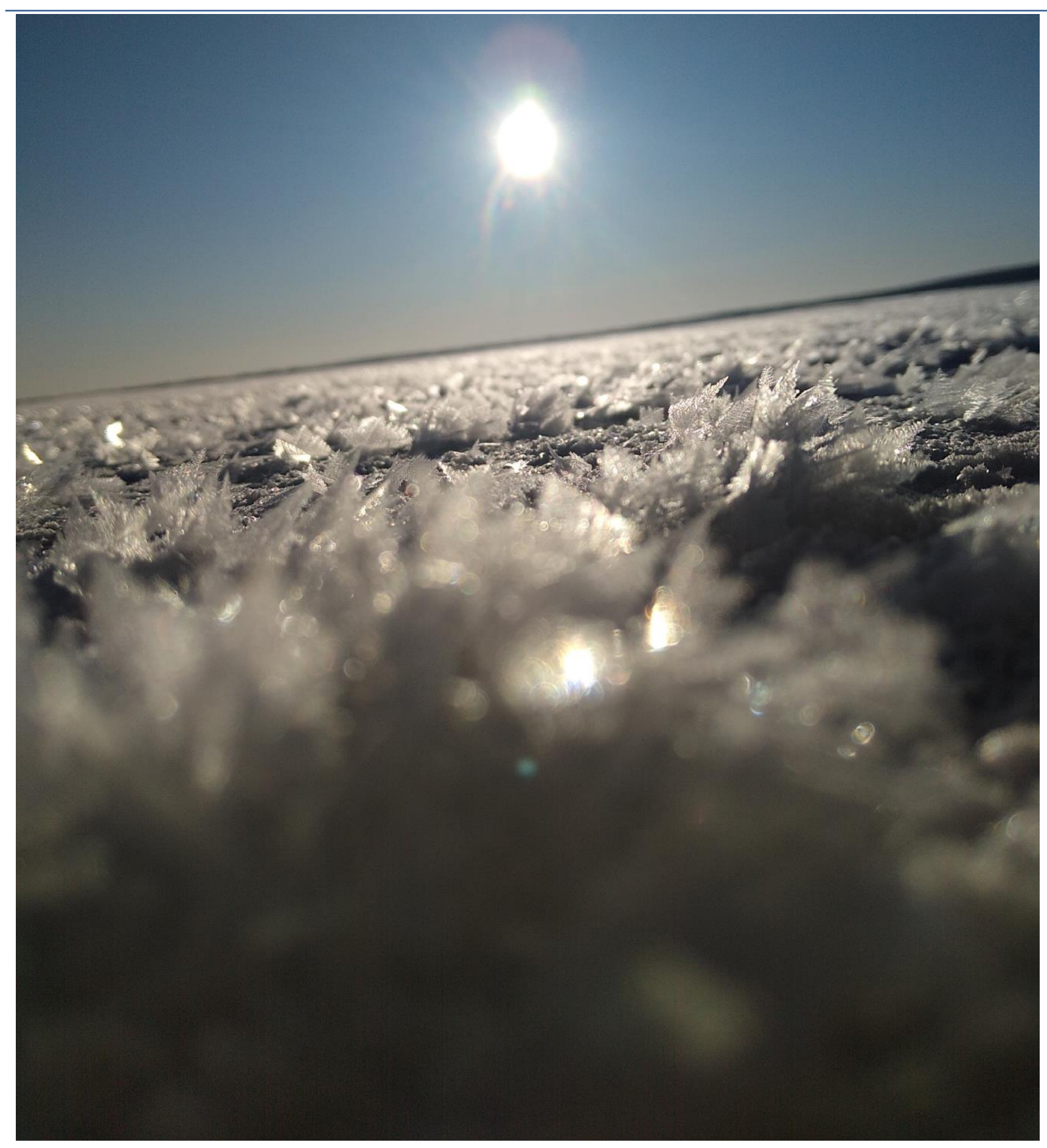

Sunset at Lake Higgins in Northern Michigan, USA, rated by National Geographic as the $6^{\text {th }}$ most beautiful lake in the world. Dan Hermes, Michigan, USA. 